\title{
CAUSAS DE LA FALTA DE ASISTENCIA A CLASE EN UNA FACULTAD DE CIENCIAS: ANÁLISIS DE LOS RESULTADOS DE ENCUESTAS PERSONALES
}

\author{
CAUSES FOR THE STUDENT'S ABSENCE IN A SCIENCE FACULTY. \\ ANALYSIS OF THE RESULTS FROM PERSONAL INQUIRIES
}

\author{
José Ángel Álvarez Saura*, Esther Duque Casas**, Manuel García Basallote***, \\ $M^{a}$ Valme García Moreno****, Concepción García Vázquez*****, \\ Ignacio de Ory Arriaga****** e Irene Rodríguez Blázquez** \\ Grupo de Acción Tutorial de la Facultad de Ciencias de la Universidad de Cádiz
} Facultad de Ciencias. Puerto Real (Cádiz)

\section{RESUMEN}

Se realizó una completa serie de encuestas personales a alumnos de la Facultad de Ciencias de la Universidad de Cádiz, con el objeto de conocer las causas por las que dejan de asistir a las clases presenciales. El estudio realizado permite hacer un análisis detallado de los motivos apuntados por el conjunto de la población encuestada así como discriminar un grupo especial de alumnos de baja asistencia, con particularidades significativas.

Palabras clave: Tutorización universitaria, absentismo, encuestas.

Grupo de Acción Tutorial de la Facultad de Ciencias de la Universidad de Cádiz.

( $2^{\circ}$ Premio en la I convocatoria de Acciones de Mejora del Consejo de Universidades y la Secretaría General del MEC y D., año 2000).

* José Ángel Álvarez Saura es Profesor Asociado de la UCA, Dpto. de Química Física.

** Esther Duque Casas e Irene Rodríguez Blázquez son alumnas becarias de la UCA y del Instituto Andaluz de la Juventud asignados al grupo de Acción Tutorial.

*** Manuel García Vasallote es Profesor Titular de la UCA, Dpto. de Ciencia de los Materiales e ingeniería Metalúrgica y Química Inorgánica.

**** $\mathrm{M}^{\mathrm{a}}$ Valme García Moreno es Profesor Asociado de la UCA, Dpto. de Química Analítica.

***** Concepción García Vázquez es Profesora Asociada de la UCA, Dpto. de Matemáticas.

****** Ignacio de Ory Arriaga es Profesor Asociado de la UCA, Dpto. de Ingeniería Química, Tecnología de Alimentos y Tecnología del Medio Ambiente. 


\begin{abstract}
A complete personal inquiry were carried out to know the real causes for students from a Science Faculty (University of Cádiz) to fail attending their classes. The current study allows to analyze the causes pointed by the general group as well as to distinguish a particular group of students classified as low attendance.
\end{abstract}

Keywords: Tutorisation, absenteeism, poll.

\title{
Introducción
}

A partir del Curso Académico 1998/99 se puso en práctica en la Facultad de Ciencias de la Universidad de Cádiz un Programa para la tutorización de alumnos universitarios que se ha dado a conocer como Proyecto Brújula. Surgido a raíz del proceso de evaluación del Título de Licenciado en Químicas de dicha Universidad en 1996, este proyecto obtuvo el $2^{\circ}$ Premio en la I Convocatoria de Acciones de Mejora del Consejo de Universidades y la Secretaría General del Ministerio de Educación, Cultura y Deporte (2000). Básicamente, consiste en un programa de orientación para alumnos universitarios de nuevo ingreso cuyos objetivos principales son:

- Informarles desde una perspectiva más cercana de sus opciones para confeccionar el currículum.

- Realizar un seguimiento a través de entrevistas concertadas de su grado de aprovechamiento académico.

- Conocer y valorar los problemas que los estudiantes aprecian a la hora de seguir dentro de un marco de normalidad los estudios de su Titulación.

El Programa ha sido aplicado a partir de entonces con un notable éxito, encontrándose entre las principales actividades ya realizadas las siguientes:

- Realización de Seminarios específicos relacionados con el creditaje, planes de estudio, normativa de matriculación, optatividad, libre elección, etc.

- Asignación de un Profesor Tutor a cada alumno de nuevo ingreso para una orientación personalizada.

- Apertura y puesta en marcha de una Oficina permanente de Atención al Alumno, despachada por becarios de últimos cursos de las distintas Titulaciones.

- Realización de encuestas para recoger la opinión de los alumnos sobre distintos aspectos del funcionamiento académico.

Es precisamente dentro de este último apartado donde se inserta el estudio recogido en el presente trabajo, realizado durante el Curso Académico 2000/2001, y que pretende conocer las causas de la no asistencia a clases por parte de los alumnos. Esta problemática preocupa seriamente al profesorado de la Facultad, ya que afecta a un porcentaje considerable de los alumnos matriculados, aunque puede considerarse que se trata de un problema bastante extendido en las demás Titulaciones de la UCA y de otras Universidades españolas.

A continuación, se detallan los aspectos más relevantes del diseño realizado para dicha encuesta así como algunas particularidades sobre el ámbito de aplicación de la misma. 


\section{Metodología}

La Facultad de Ciencias de la Universidad de Cádiz ofertó en el Curso 2000/01 tres Titulaciones superiores: Ingeniero Químico, Licenciado en Matemáticas y Licenciado en Químicas. Sobre un universo total de 1600 alumnos matriculados en dichas Titulaciones (de 5 Cursos cada una, excepto Matemáticas cuya $1^{\mathrm{a}}$ promoción todavía cursaba $4^{\circ}$ ) se realizó una encuesta personal entre los días 19 y 20 de Marzo de 2001, días lectivos sin incidencias particulares que afectaran a la asistencia. El procedimiento elegido fue la entrega de un formulario (que se detallará más adelante) a cada uno de los alumnos presentes durante una hora de clases no anunciada previamente. El número de alumnos encuestados en las aulas se detalla en la tabla 1 .

TABLA 1: Número de alumnos encuestados en el aula por Titulación.

\begin{tabular}{|l|c|c|c|c|c|c|}
\hline \multicolumn{1}{|c|}{ Titulación\Curso } & $\mathbf{1}^{\mathbf{0}}$ & $\mathbf{2}^{\mathbf{o}}$ & $\mathbf{3}^{\mathbf{0}}$ & $\mathbf{4}^{\mathbf{0}}$ & $\mathbf{5}^{\mathbf{0}}$ & Total \\
\hline Ingeniero Químico & 69 & 38 & 43 & 54 & 48 & $\mathbf{2 5 2}$ \\
\hline Matemáticas & 76 & 27 & 30 & 21 & - & $\mathbf{1 5 4}$ \\
\hline Química & 82 & 75 & 39 & 60 & 43 & $\mathbf{2 9 9}$ \\
\hline Total de la Facultad & \multicolumn{7}{|l}{} & $\mathbf{7 0 5}$ \\
\hline
\end{tabular}

Conscientes de que para una encuesta cuyo objetivo sea analizar las causas de no asistencia a clases tiene un interés especial analizar las opiniones de aquellos alumnos matriculados que no asisten regularmente al aula, se remitió un modelo idéntico al original al domicilio de un número dado de alumnos seleccionados al azar, adjuntando un sobre franqueado para que pudieran devolver la encuesta cumplimentada al Grupo de Acción Tutorial.

Se seleccionaron al azar un total de 280 alumnos de la Facultad, tomando 20 alumnos de cada curso y titulación. Para evitar la duplicación de datos, se pidió que indicaran si ya habían cumplimentado en la encuesta en el aula. El número total de respuestas recibidas fue de 41, de las cuales tan sólo 20 fueron de alumnos no encuestados ya en el aula. Dado que el reducido número de encuestas válidas obtenidas por esta vía hacía poco representativo este estudio, se decidió diseñar un nuevo estudio discriminatorio a partir de las encuestas realizadas en el aula, seleccionando aquellas en las que el alumno registra una baja asistencia a clases, según el siguiente criterio:

- Se seleccionan aquellos alumnos que en la cuestión 3 (¿cuál sería en conjunto tu nivel de asistencia?) han señalado la opción 1 (menos del 25\%) o la opción 2 (entre el 25 y el 50\%).

- Se seleccionan aquellos alumnos en los que el cociente entre la respuesta a la cuestión 2 (¿de cuántas asignaturas asistes al menos al 50\% de las clases?) y la respuesta a la cuestión 1 (señala el número de asignaturas en las que estás matriculado) resulta menor que $1 / 2$.

El número total de alumnos seleccionados por estos criterios se recoge en la tabla 2. 


\section{TABLA 2: Número de alumnos considerados de baja asistencia de entre de los encuestados en al aula.}

\begin{tabular}{|l|c|c|c|c|c|c|}
\hline Titulación\Curso & $\mathbf{1}^{\mathbf{0}}$ & $\mathbf{2}^{\mathbf{o}}$ & $\mathbf{3}^{\mathbf{o}}$ & $\mathbf{4}^{\mathbf{0}}$ & $\mathbf{5}^{\mathbf{0}}$ & Total \\
\hline Ingeniero Químico & $1(1.5 \%)$ & $2(5.3 \%)$ & $6(14 \%)$ & $7(13 \%)$ & $12(25 \%)$ & $\mathbf{2 8}(\mathbf{1 1 . 1 \%})$ \\
\hline Matemáticas & $4(5.3 \%)$ & $4(14.8 \%)$ & $1(3.3 \%)$ & $1(4.8 \%)$ & - & $\mathbf{1 0}(\mathbf{6 . 5 \% )}$ \\
\hline Química & $10(12.2 \%)$ & $13(17.3 \%)$ & $9(23.1 \%)$ & $3(5 \%)$ & $6(14 \%)$ & $\mathbf{4 1}(\mathbf{1 3 . 7 \% )})$ \\
\hline Total de la Facultad & & & & & & $\mathbf{7 9}(\mathbf{1 1 . 2 \% )}$ \\
\hline
\end{tabular}

En el Anexo I se muestra el formulario completo entregado en el aula y enviado a domicilio. Se trata de 6 cuestiones generales, una tabla de causas de no asistencia a clase con valoración de importancia, una cuestión resumen de causas y unas propuestas del alumno para mejorar la asistencia.

\section{Resultados}

El conjunto de datos obtenidos y la discusión de sus resultados han sido agrupados en dos grandes apartados en función del origen de las encuestas: Encuestas presenciales en general y Alumnos de menor asistencia en el que se destacarán, a partir de las encuestas del primer apartado (presenciales) y tal como se explicó en la metodología, aquellas que pertenecen a alumnos que declaran tener una asistencia por debajo del 50\% (ver apartado 3.2).

Dentro de cada apartado se analizarán separadamente las cuestiones generales (cuestiones 1-6) y las causas de no asistencia (pregunta 7). Por último, se analizarán las sugerencias aportadas por los alumnos (pregunta 8).

\section{Encuestas presenciales}

Se recogen aquí los resultados de las encuestas realizadas en el aula al número de alumnos detallado en la tabla 1. En su conjunto, puede considerarse que las opiniones manifestadas por estos alumnos son representativas del conjunto de los alumnos que suele asistir a clase. Para el análisis de resultados se tendrán en cuenta los distintos apartados de la encuesta.

\section{Cuestiones generales}

En las cuestiones 1 y 2 se preguntaba al alumno sobre el número de asignaturas en las que está matriculado y el número de asignaturas a las que asiste a más de la mitad de las clases. Con los datos de todas las encuestas se han calculado los valores promedio.

Los resultados obtenidos para toda la Facultad, que han sido esquematizados en la figura 1 , muestran que los alumnos se matriculan de una media de 10.55 asignaturas, mientras que asisten regularmente tan sólo a 8.1 asignaturas, lo que supone un $76.74 \%$ sobre el total. 


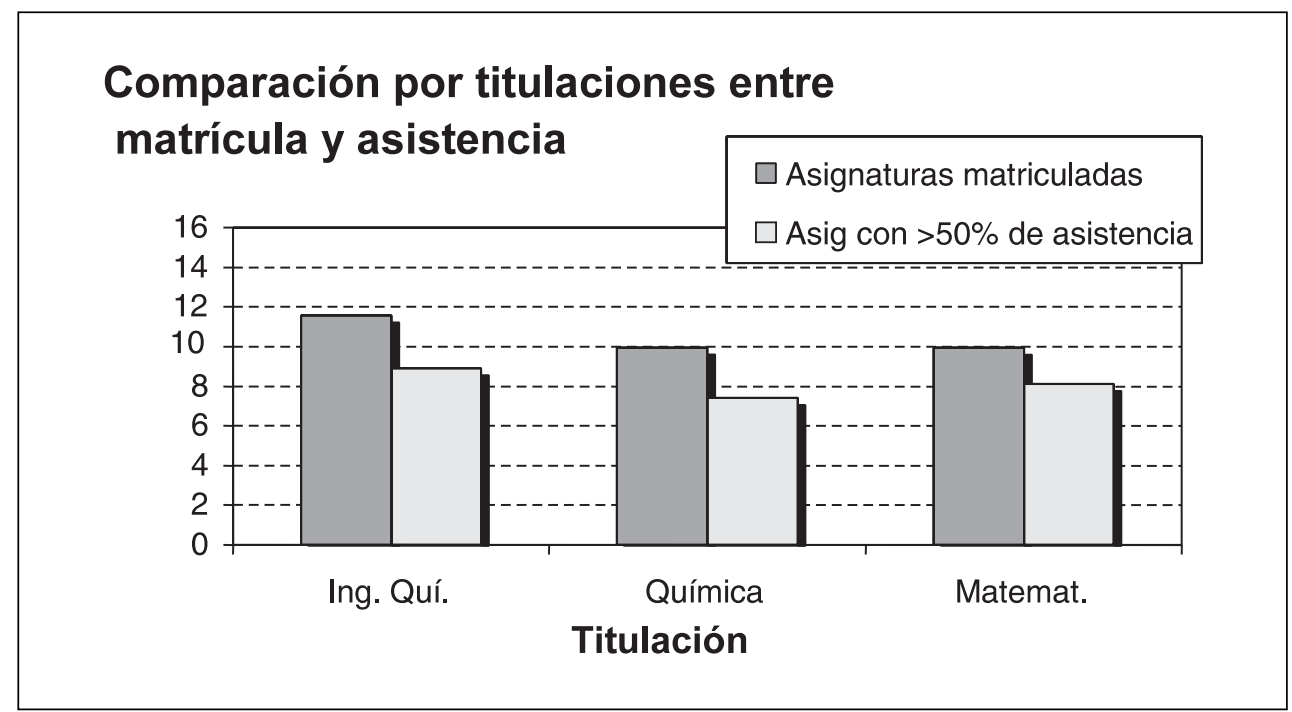

FIGURA 1.

Respuestas a las cuestiones 1 y 2 (Conjunto de la Facultad).

Por titulaciones, la que mayor porcentaje de absentismo medio presenta es Químicas (asisten a un $74.24 \%$ de las asignaturas matriculadas), seguido de Ingeniero Químico $(77.13 \%)$ y por último Matemáticas $(81.78 \%)$.

Aunque los datos estudiados por cursos no se han representado aquí, es interesante apuntar que en Ingeniero Químico se detecta una disminución de la asistencia a clase a medida que se avanza hacia los cursos superiores. En Matemáticas y Química la tendencia no es tan clara, observándose una disminución de la asistencia entre $1^{\circ}$ y $2^{\circ}$, manteniéndose con altibajos en los cursos siguientes.

La cuestión número 3 pedía a los alumnos que especificaran su nivel de asistencia general a clase. Los resultados obtenidos (Fig. 2) muestran que aproximadamente un $75 \%$ de todos los alumnos encuestados afirma que su asistencia se sitúa por encima del $75 \%$ de las clases; para un $20 \%$ de los alumnos la asistencia está entre el $50-75 \%$, y tan sólo un 5\% afirma asistir a menos del $50 \%$. Estos porcentajes se mantienen prácticamente iguales si analizamos los datos por titulación y por cursos.

La cuestión 4 pedía al alumno que señalara si el tipo de asignatura (Troncal u Obligatoria, Optativa, Libre Elección o Nivelación) influye sobre su nivel de asistencia a clase, y en caso afirmativo indicara a qué tipos de asignatura asiste con menos frecuencia. Los resultados se muestran en la figura 3 .

Entre el 60 y el 75\% de los encuestados, según la Titulación que curse, afirma que el tipo de asignatura no influye en su asistencia a clase, siendo los alumnos de Matemáticas los más indiferentes. Entre los alumnos a los que sí afecta el tipo de asignatura en su nivel de asistencia, se observa que, por este orden, faltan más a asignaturas de Libre Elección, seguido de Optativas y por último, Troncales y Obligatorias. 


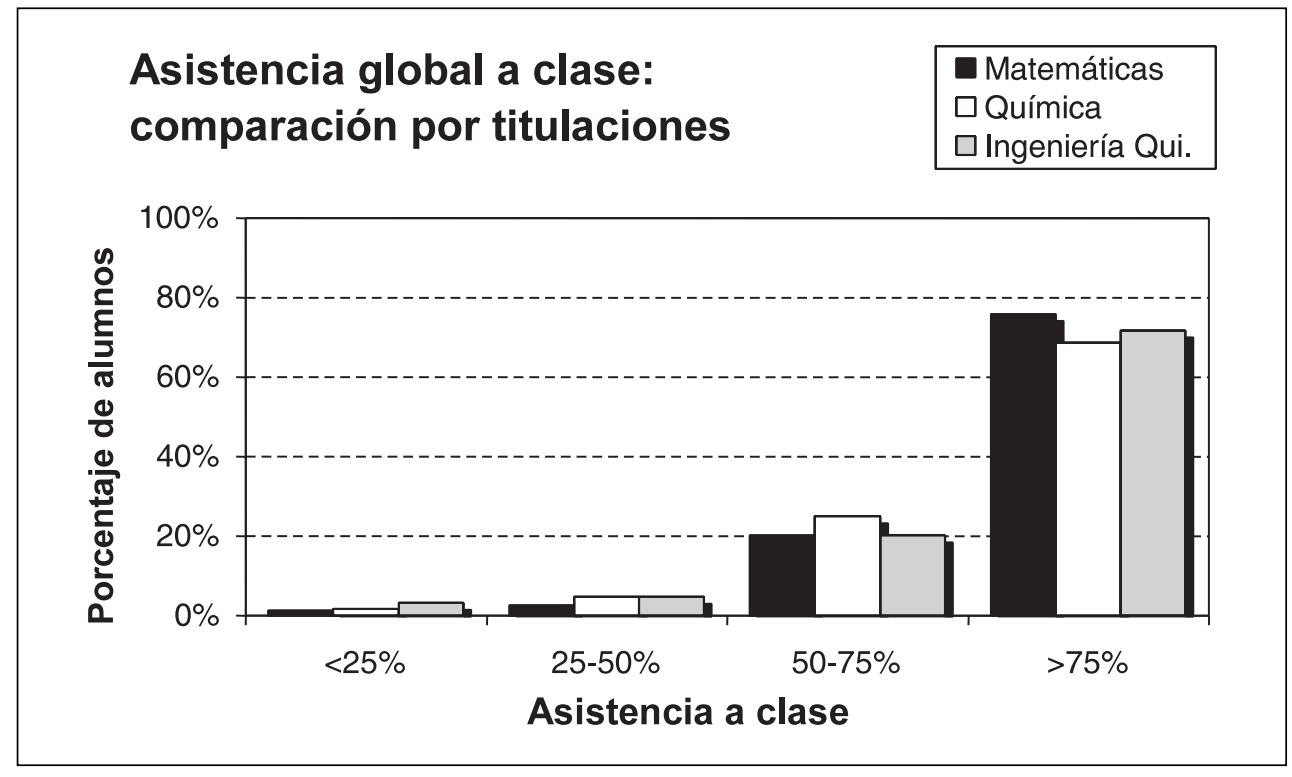

FIGURA 2.

Respuestas a la cuestión 3 (Conjunto de la Facultad).

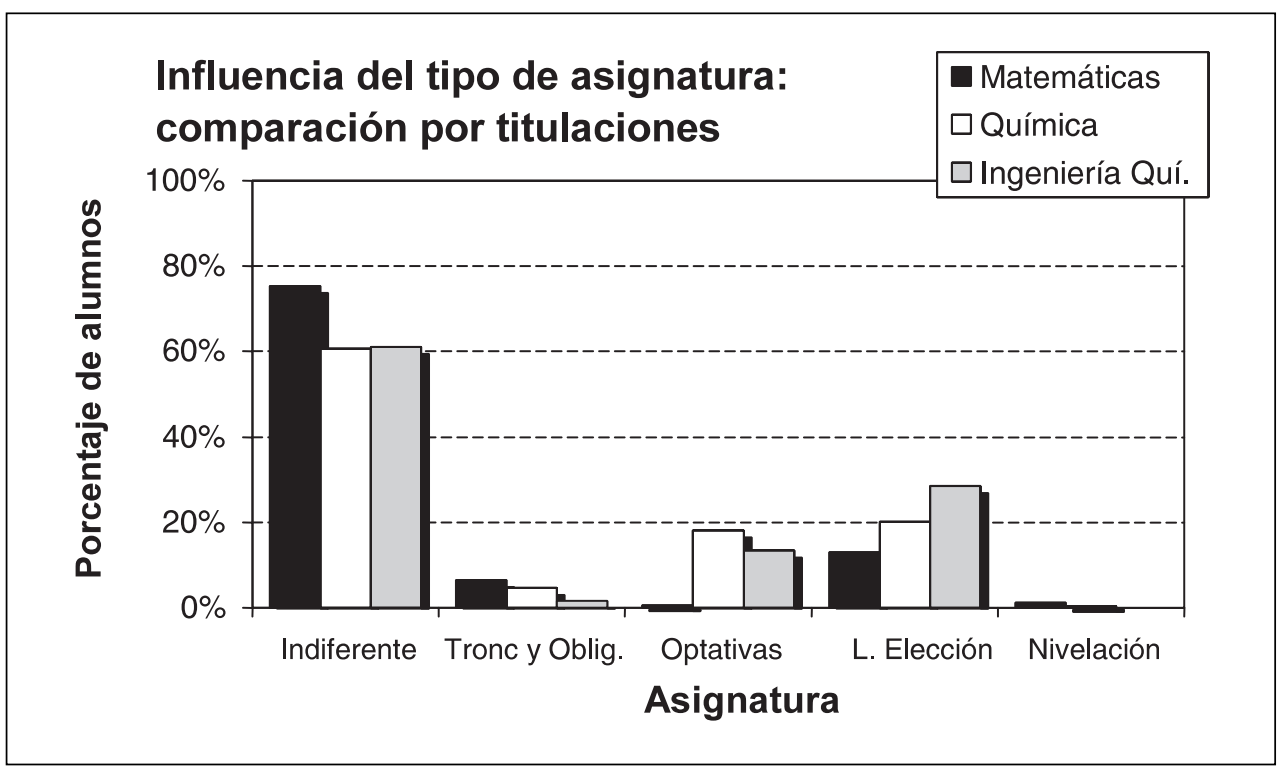

FIGURA 3.

Respuestas a la cuestión 4 (Conjunto de la Facultad). 
En la cuestión 5 se pregunta al alumno si trabaja (ya sea temporalmente o todo el año) (ver Fig. 4). En el total de la Facultad, un $75 \%$ de los alumnos señalaron que no trabaja, el $19 \%$ que trabaja temporalmente, y el $6 \%$ que trabaja todo el año. El porcentaje más alto de alumnos que trabaja durante todo el año corresponde a Matemáticas (11\%), y el más bajo a Ingeniero Químico (2\%).

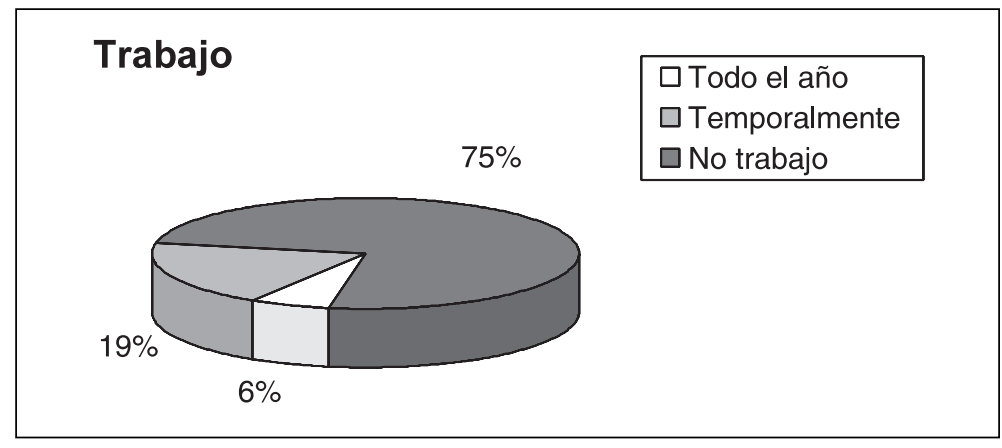

FIGURA 4.

Respuestas a la cuestión 5 (Conjunto de la Facultad).

La cuestión 6 se refiere a la condición de becario del alumno. En la figura 5 se observa que el $28 \%$ de los alumnos encuestados recibe beca, mientras que el $67 \%$ de los alumnos no disfruta de ayuda económica suplementaria.

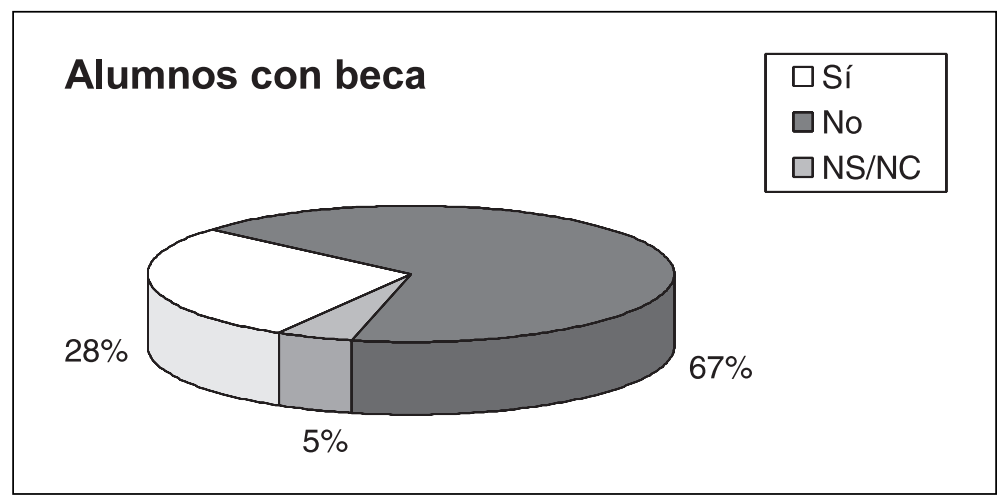

FIGURA 5.

Respuestas a la cuestión 6 (Conjunto de la Facultad).

\section{Causas de absentismo}

Como se indicó en la encuesta, los alumnos tenían la posibilidad de puntuar el nivel de influencia de cada una de las posibles causas de falta de asistencia en una escala de 0 (nada) a 4 (mucho). Aunque del conjunto de datos obtenidos podría derivarse un análisis mucho 
más detallado de las causas principales por tipo de Titulación y Curso, el aquí realizado se basa en la puntuación media otorgada por todos los alumnos (a nivel del conjunto de la Facultad) y que ha sido esquematizada en la figura 6 . A continuación, se destacarán las principales causas apuntadas.

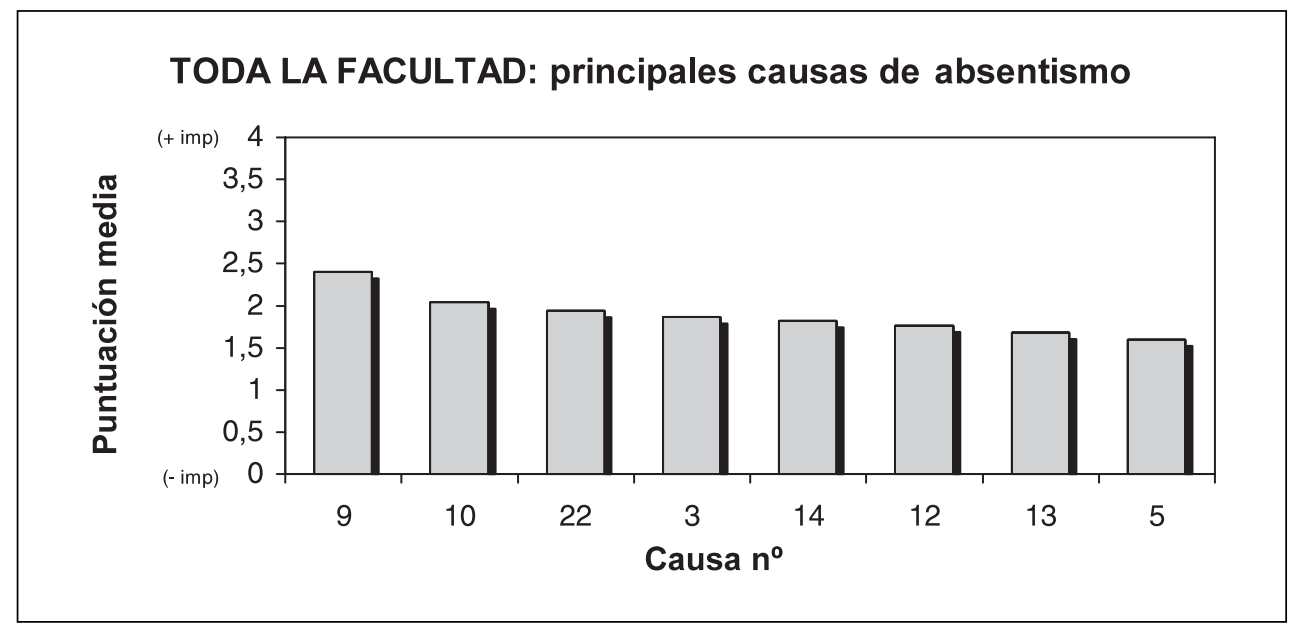

FIGURA 6.

Respuestas a la cuestión de Causas (Conjunto de la Facultad).

La causa más importante por la que los alumnos dicen faltar a clase es que el profesor no explica de manera clara y estructurada (causa 9), que ha obtenido una puntuación de 2,40 . Este dato es debido sobre todo a la elevada valoración otorgada por alumnos de los primeros cursos de todas las titulaciones, (en los que además el número de encuestados es más elevado), perdiendo importancia en cursos superiores.

La siguiente en importancia es suelo no asistir a clase los días antes de un examen (causa 10). En tercera posición para el total de la Facultad se sitúa las clases resultan aburridas (causa 22). La cuarta causa más valorada para el total de la Facultad es suelo faltar a aquellas clases sueltas en el horario (causa 3), aunque es menos apuntada por los alumnos de matemáticas. La clase me coincide con otras asignaturas teóricas (causa 14) aparece en quinto lugar. Le sigue en importancia la asignatura (por ejemplo de Libre Elección) se imparte en otro centro (causa 12).

Algunas particularidades significativas de las distintas titulaciones son apuntadas a continuación:

- en Matemáticas, la causa las clases resultan aburridas (causa 22) adquiere mayor importancia que en el resto de Titulaciones ( $2^{\mathrm{a}}$ en importancia). También aparece como causa muy importante ( $3^{\text {er }}$ puesto) que el nivel de conocimientos necesarios para seguir la clase es muy alto (causa 1) y, sin embargo, parece no tener apenas trascendencia en el resto de las titulaciones pues aparecen en los puestos $22^{\circ}$ (Químicas) y $24^{\circ}$ (Ingeniero Químico). Del mismo modo, el motivo dejo alguna asignatura porque me he dado cuenta de que me he matriculado de más asignaturas de las que puedo apro- 
bar (causa 24) también es más importante en Matemáticas que en el resto de las titulaciones ( $6^{\circ}$ lugar).

- en Ingeniero Químico, la causa el número de horas es excesivo y me canso (causa 4) ocupa la $7^{\mathrm{a}}$ posición, mientras que en Química aparece en $14^{\circ}$ lugar y en Matemáticas en $13^{\text {er }}$ lugar. Se recordará aquí que el promedio de asignaturas matriculadas para los alumnos de Ingeniero Químico supera, en más de un punto y medio, a los promedios de Matemáticas y Químicas (Fig. 1).

- en Químicas, dispones de apuntes de otros compañeros (causa 13) ocupa la $5^{\mathrm{a}}$ posición, y en cambio, aparece mucho más atrás en Ingeniero Químico y en Matemáticas.

Entre las causas menos apuntadas aparecen, con un promedio inferior a 0,5 puntos, las siguientes:

- No puedes asistir a clase por tu trabajo o por estar realizando el servicio militar o la prestación social sustitutoria.

- Estoy matriculado en esta titulación, pero realmente no me gusta.

- No sé tomar apuntes.

\section{Sugerencias}

El último apartado del formulario de esta encuesta, pedía al alumno que propusiera medidas que mejorasen su propio nivel de asistencia. La pregunta es muy abierta lo que supone, a la hora de tabular los datos, la dificultad de agrupar bajo un mismo enunciado propuestas expresadas de manera distinta.

La propuesta que se repite con mayor frecuencia en todos los cursos de todas las titulaciones es la de mejorar la metodología de los profesores. Incluso en los cursos superiores (en los que las causas relacionadas con la labor del profesorado pierden bastante importancia con respecto a otras) esta propuesta supera ampliamente a las demás.

Otra sugerencia que se repite con bastante frecuencia es mejorar el transporte público, a pesar de que la causa falta involuntaria por problemas de transporte no es de las más valoradas (aparece en el puesto $11^{\circ}$ en el total de las encuestas realizadas en el aula).

Finalmente, las propuestas relacionadas con la mejora de horarios son también frecuentes, sobre todo referidas a evitar horas sueltas en el horario o evitar el solapamiento de clases.

\section{Alumnos de menor asistencia}

De los dos criterios de selección de encuestas en el aula descritos en la metodología, se seleccionaron aquellos alumnos que cumplieran al menos uno de los dos señalados. El resultado, como se observa en la tabla 2 , es que se seleccionaron el $11,2 \%$ de las encuestas de aula (79 alumnos).

Al igual que se hizo en el apartado 3.1, se dividirá la discusión de los resultados obtenidos para esta población de alumnos en función del apartado de la encuesta. 


\section{Cuestiones generales}

En la figura 7, resumen de las cuestiones 1 y 2, puede observarse que, para el conjunto de las tres titulaciones, el promedio de asignaturas en las que están matriculados los alumnos de menor asistencia es ligeramente superior al número de asignaturas correspondientes al total de alumnos encuestados (10.96 asignaturas en los alumnos de menor asistencia frente a 10.55 asignaturas en el total de alumnos). Asimismo, la asistencia (promedio de asignaturas a las que el alumno asiste más del $50 \%$ ) se reduce a más de la mitad del promedio general (3,85 asignaturas en los alumnos de menor asistencia frente a 8,10 asignaturas en el total de alumnos).

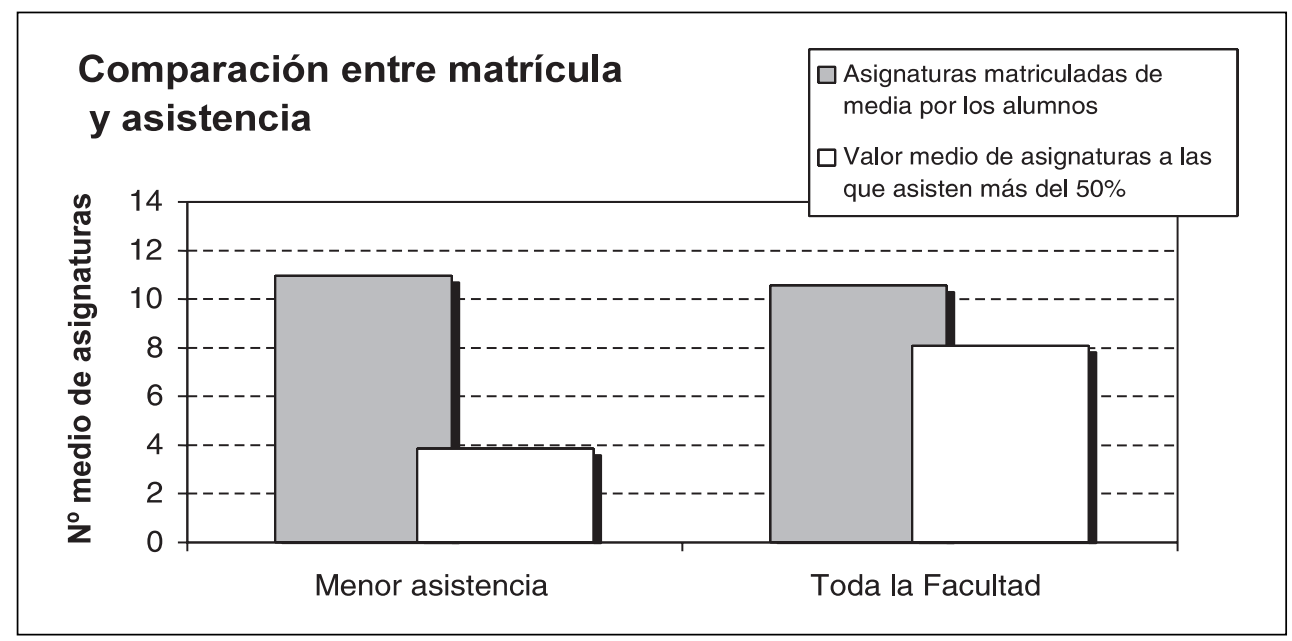

FIGURA 7.

Respuestas a las cuestiones 1 y 2 (Menor Aistencia vs. Toda la Facultad).

En la figura 8 se esquematizan las respuestas a la cuestión 3, dedicada al nivel de asistencia media del alumno. Se detecta un sensible porcentaje (22\%) de estos alumnos identificados como de baja asistencia que afirman acudir a más del $75 \%$ de las clases, lo que demuestra cierta incoherencia entre las respuestas 1,2 y 3 .

En cuanto a la cuestión 4 (ver Fig. 9) el porcentaje de alumnos que afirma que el tipo de asignatura no influye en su nivel de asistencia se mantiene próximo al $60 \%$, siendo esta cifra ligeramente inferior a la correspondiente al total de alumnos encuestados. Al igual que en 3.1, los alumnos de Matemáticas son los que se muestran más indiferentes al tipo de asignatura. Entre los alumnos a los que sí afecta, se observa que faltan más, por este orden, a Libre Elección, seguido de Optativas y por último, Troncales y Obligatorias.

Sobre las cuestiones 5 y 6 , cuyos resultados se esquematizan en las figuras 10 y 11, se observa que aumentan sensiblemente los porcentajes de alumnos que trabajan tanto temporalmente como todo el año, lo que explicaría en parte el bajo nivel de asistencia a clases registrado en esta fracción de población. En cambio, disminuye a casi la mitad la proporción de alumnos con beca. 


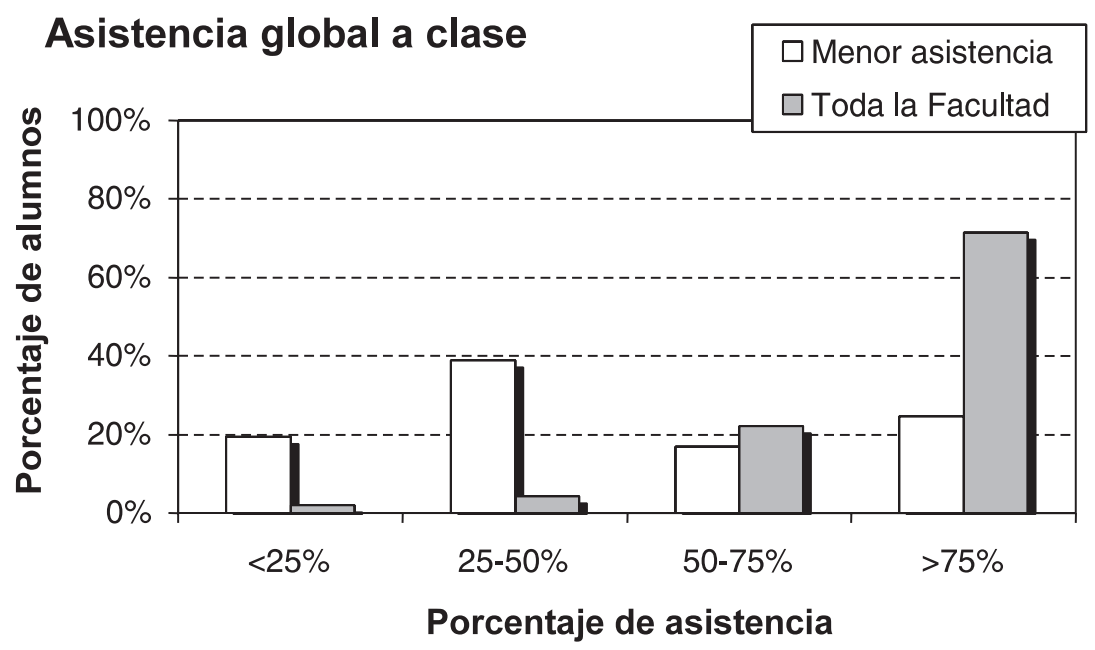

FIGURA 8.

Respuestas a la cuestión 3 (Menor Asistencia vs. Toda la Facultad).

\section{Influencia del tipo de asignatura}

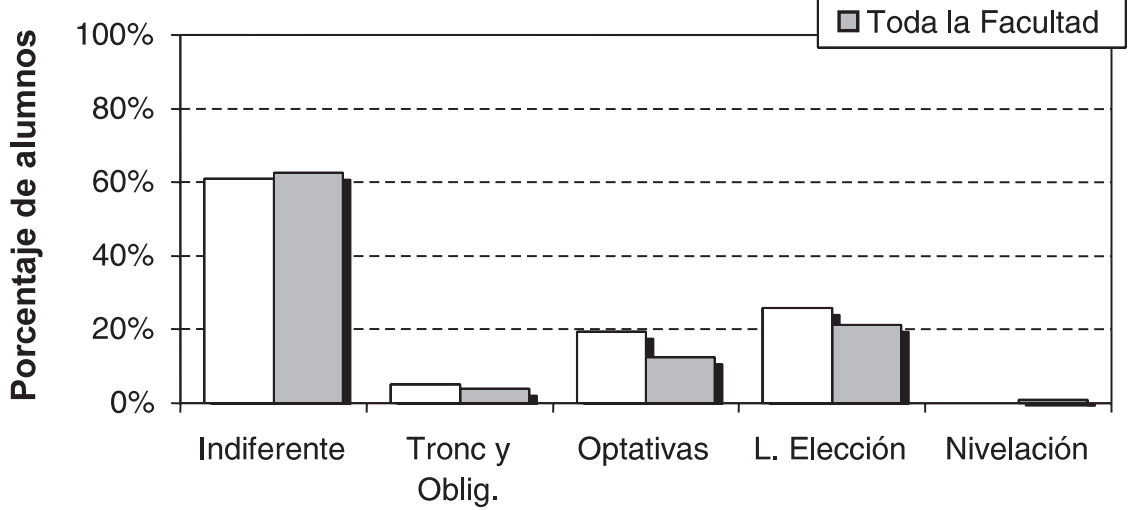

\section{Asignatura}

Respuestas a la cuestión 3 (Menor Asistencia vs. Toda la Facultad). 


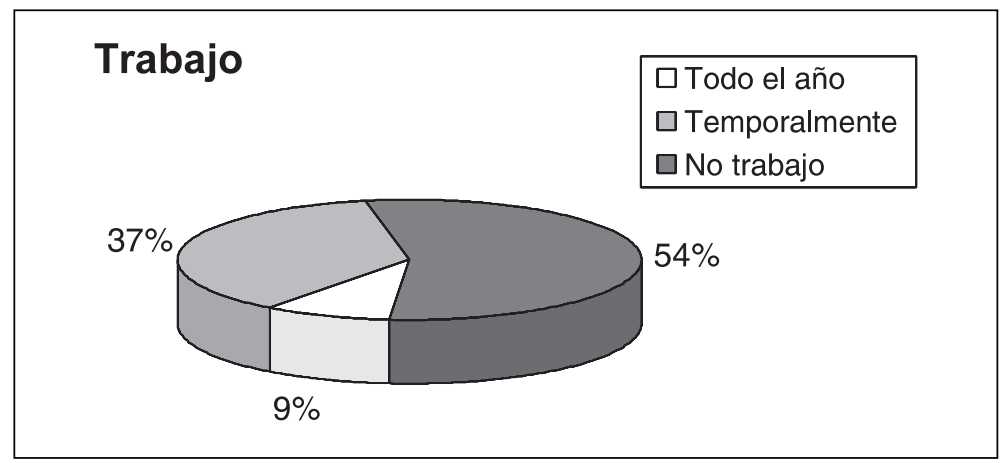

FIGURA 10.

Respuestas a la cuestión 4 (Menor Asistencia vs. Toda la Facultad).

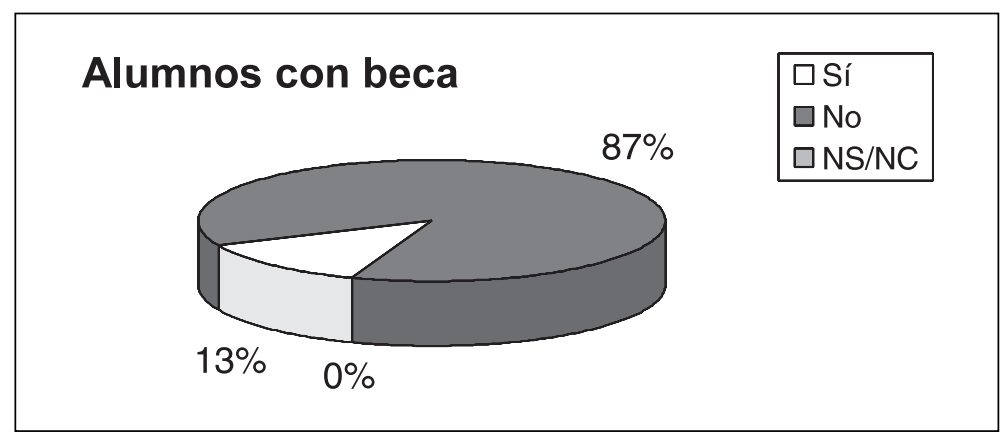

FIGURA 11.

Respuestas a la cuestión 3 (Menor Asistencia vs. Toda la Facultad).

\section{Causas de absentismo}

Las causas que motivan la falta de asistencia a clase de este grupo de alumnos se pueden esquematizar en la figura 12, y son, como se verá, distintas respecto a las señaladas por el global de los alumnos.

La primera causa apuntada entre los alumnos que presentan una menor asistencia es suelo no asistir a clase los días antes de un examen (causa 10), con una puntuación muy elevada $(3,04)$. Esta razón no explicaría completamente, sin embargo, el bajo nivel de asistencia general de esta población, pues se refiere a ausencias muy puntuales a clase. No obstante, se puede considerar como lógica pues si este tipo de alumnos falta de una manera generalizada a clases es previsible que decida no asistir antes de los exámenes.

Otra causa que adquiere especial relevancia en este grupo es dispones de apuntes de otros compañeros (causa 13), que se sitúa en segunda posición (en el total de alumnos aparecía en $7^{\circ}$ lugar), con lo que destaca como una de las causas diferenciadoras entre los dos grupos de alumnos estudiados; parecería que éstos últimos conceden menor importancia a 


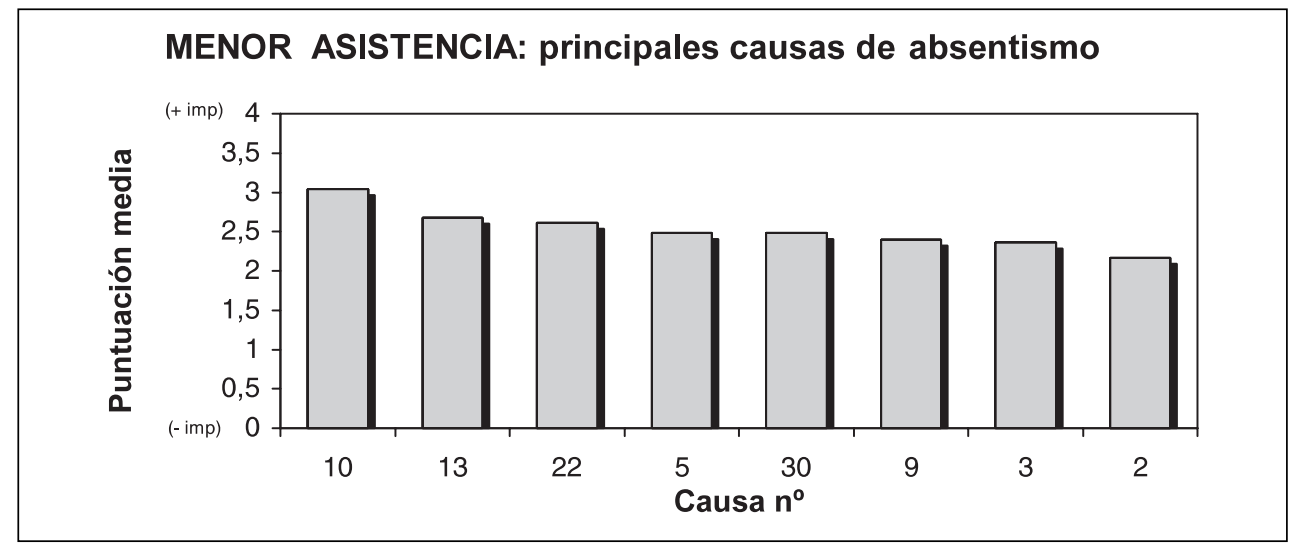

FIGURA 12.

Respuestas a la cuestión de Causas (Menor Asistencia vs. Toda la Facultad).

la aportación del profesor a su aprendizaje o quizá que no se reconocen capaces de aprovechar correctamente una clase presencial.

En esta línea, como $3^{\text {a }}$ causa aparece las clases resultan aburridas (causa 22) seguida de tengo la asignatura pendiente y no necesito seguir las clases (causa 5), lo que permite confirmar esta conclusión.

Dejo de asistir en fechas concretas (vísperas de periodos de exámenes, vacacionales, etc.) (causa 30), pasa a ser la quinta en este grupo (anteriormente aparecía como $9^{\mathrm{a}}$ causa).

En cambio, pierden importancia las siguientes causas: el profesor no explica de manera clara y estructurada (causa 9), que pasa a ocupar la sexta posición (antes aparecía en primer lugar), suelo faltar a aquellas clases sueltas en el horario (causa 3), que se sitúa como la séptima (antes la 4a), y la clase me coincide con otras asignaturas teóricas (causa 14).

Como resumen, en este grupo se refuerzan aquellas causas que tienen más que ver con una decisión personal del alumno de no asistir a clase, empleando su tiempo de manera que él puede considerar más provechosa: suelo no asistir a clase los días antes de un examen, dispongo de apuntes de otros compañeros, tengo la asignatura pendiente y no necesito seguir las clases, etc. La excepción se da, de nuevo, en Matemáticas, donde las causas más importantes hacen referencia fundamentalmente a la forma en que se imparten las clases: el profesor no explica de manera clara y estructurada (9), o las clases resultan aburridas (22).

Las causas menos apuntadas son, en general, coincidentes con las obtenidas para el total de alumnos.

\section{Conclusiones}

1. Para la población estudiada, se pueden diferenciar claramente dos tipos de alumnos en lo que respecta a la asistencia a clases: 
- Un primer grupo, que en el presente estudio estaría constituido por aproximadamente el $85 \%$ de los encuestados, asiste con relativa frecuencia a las aulas (siempre por encima del $50 \%$ de las clases presenciales), aunque los datos demuestran que de manera regular sólo lo hace a una media de 8 asignaturas sobre las 10 en que se matricula, lo que sugiere que no se sienten completamente capaces de abordar toda la carga lectiva escogida. Para estos alumnos, las principales causas que les hacen faltar a clase son aquellas relacionadas con la labor docente del profesor o bien circunstancias coyunturales como que dejan de asistir en los días previos a los exámenes.

- Un segundo grupo, constituido por el 10-15\% de los alumnos consultados, reconoce asistir a menos del 50\% del total de las clases con lo que pueden ser considerados como alumnos de baja asistencia. Además, de una media de 11 asignaturas en las que se halla matriculado tan sólo asiste con cierta regularidad a 3 ó 4 . Para este grupo, los motivos de sus frecuentes ausencias a clase son más de índole personal, decisiones conscientes adoptadas porque disponen de apuntes de otros compañeros, porque dicen aburrirse en clase o porque son alumnos repetidores de asignaturas y no consideran necesario volver a asistir al aula y deciden emplear su tiempo de la manera que pueden considerar más provechosa. En este grupo, la cercanía de exámenes es también un motivo decisivo para dejar de asistir a clase.

- Existe un tercer grupo formado por los alumnos que no han cumplimentado la encuesta en el aula. Es imposible establecer una valoración objetiva de sus opiniones, ya que el nivel de respuestas a la encuesta por correo también fue escasa. En cualquier caso, puede considerarse que su opinión debe ser próxima a la de los alumnos del segundo grupo mencionado arriba (menor asistencia).

2. Las titulaciones de Químicas e Ingeniero Químico presentan un perfil bastante similar en cuanto a causas que motivan la falta de asistencia, adquiriendo la mayor importancia la causa 9 (el profesor no explica de manera clara y estructurada) y, tras ella, otros motivos que tienen más que ver con una decisión personal del alumno (suelo no asistir a clase los días antes de un examen, suelo faltar a aquellas clases sueltas en el horario, etc.). En cambio, en Matemáticas las causas hacen más hincapié en la percepción del alumno sobre cómo se imparten las clases (las clases resultan aburridas, el nivel de conocimientos necesarios para seguir la clase es muy alto).

4. Aunque no es el objetivo del presente trabajo, otra conclusión de interés se refiere a los porcentajes de absentismo en función de la Titulación en que se esté matriculado; los alumnos de Ciencias Químicas e Ingeniero Químico tienen un porcentaje sensiblemente mayor de alumnos calificados como de baja asistencia. También es reseñable cómo en Ingeniero Químico el porcentaje de alumnos de baja asistencia aumenta conforme se avanza en la titulación.

Estas conclusiones merecerían ligeras modificaciones en función del tipo de Titulación en que se esté matriculado o el nivel que se esté cursando pero, como generalización, pueden dar claves interesantes sobres las medidas a adoptar para mejorar los índices de asistencia a clases de los alumnos universitarios. Las líneas de trabajo sobre las que deberían trabajarse las acciones correctoras se proponen a continuación: 
- Según se deduce de los resultados de la encuesta, cabe esperar que una mejora en la manera de plantear y desarrollar las clases por parte del profesor resulte en un aumento de los niveles de asistencia a clase entre los alumnos que ya acuden a clase con mayor asiduidad. En este sentido, y a modo de experiencia, en el curso 2001/02 se puso en marcha en la Facultad de Ciencias de la UCA un programa de Potenciación de las Enseñanzas Prácticas (PEP) que pretende incentivar la asistencia y participación de los alumnos en clase, dedicando más tiempo y un mayor esfuerzo a la resolución de cuestiones prácticas en seminarios donde se fomente el diálogo profesor-alumno y entre los propios alumnos.

- La adopción de medidas que eviten o disminuyan los problemas de horario y transporte también se revela como un factor clave para disminuir el absentismo de los alumnos. De particular importancia es el problema relacionado con los horarios, ya que el elevado grado de optatividad y libre elección en los actuales planes de estudio obliga en muchos casos a la elección de unos horarios excesivamente amplios y con un número importante de horas sueltas.

- Aparece, por otro lado, un amplio número de alumnos para los cuales la asistencia o no a clase no está relacionada con la planificación y desarrollo de la labor docente, sino con una actitud personal frente al proceso de enseñanza-aprendizaje. La adopción de medidas que mejoren la asistencia por parte de este grupo de alumnos requiere de mayores dosis de imaginación por parte de la comunidad universitaria.

\section{Reconocimientos}

Los autores quisieran hacer constar su sincero agradecimiento al Decanato de la Facultad de Ciencias de la Universidad de Cádiz, Vicerrectorado de Alumnos, Instituto Andaluz de la Juventud y Plan Nacional de Evaluación de la Calidad de las Universidades.

\section{Referencias bibliográficas}

Del Rincón Igea, Benito (2000). Tutorías personalizadas en la Universidad. Ediciones de la Universidad de Castilla-La Mancha.

Ministerio de Educación, Cultura y Deporte (2000). Acciones de mejora. Plan de Calidad de las Universidades, pp. 13-19.

Álvarez Saura, J. A.; García Basallote, M.; García Moreno, M. V.; García Vázquez, C.; de Ory Arriaga, I. (2002). "Proyecto Brújula-Programa Tutor: Orientación en la Facultad de Ciencias de la UCA". Revista Española de Orientación Psicopedagógica, REOP. Volumen 13, número 2, $2^{\circ}$ semestre, pp. 255-260.

Álvarez Saura, J. A.; Duque Casas, E.; García Basallote, M.; García Moreno, M. V.; García Vázquez, C.; de Ory Arriaga, I.; Rodríguez Blázquez, I. (2003). "Asignaturas de nivelación: una nueva alternativa dentro de la libre elección”. Revista Innovación Educativa. N ${ }^{o}$ 13, pp. 291-304. 


\section{ANEXO I}

Grupo de Acción Tutorial. Proyecto Brújula. Facultad de Ciencias

\section{Campus Universitario del Río San Pedro} Apartado 40, Puerto Real 11510 (Cádiz) Teléfonos: 956.016300 ext:6804

Fax: 956.016288

e-mail: tutorial@uca.es

\section{Grupo de acción tutorial - Facultad de Ciencias Encuesta sobre asistencia a clase}

Durante el presente curso, el Grupo de Acción Tutorial de la Facultad de Ciencias está centrado en conocer las causas de la baja asistencia a clase de los alumnos.

Con el propósito de mejorar el funcionamiento de la Facultad, te pedimos que rellenes el siguiente cuestionario. No te llevará más de tres minutos.

\section{TITULACIÓN:}

\section{CURSO:}

\section{CUESTIONES GENERALES}

1. Señala el número de Asignaturas en que estás matriculado durante el presente curso académico 2000/2001:

2. En el curso actual (incluyendo los dos cuatrimestres), ¿de cuántas asignaturas asistes al menos al 50\% de las clases?

3. ¿Cuál sería, en conjunto, tu nivel de asistencia a clase?

Menos de $25 \%$ de las clases

Entre $25 \%$ y $50 \%$

Entre $50 \%$ y $75 \%$

Asisto a más del $75 \%$ de las clases

4. ¿Influye en tu nivel de asistencia el tipo de asignatura (troncal, libre elección, etc.)?

NO

SÍ

En caso afirmativo, ¿a qué tipo de asignaturas faltas con más frecuencia?

Troncales y obligatorias

Optativas

Libre Elección

Asignaturas de Nivelación 
5. ¿Trabajas?

Todo el año

Temporalmente

No trabajo

6. ¿Tienes beca?

Sí

NO

\section{CAUSAS}

Piensa en aquellas asignaturas o clases a las que no has asistido con regularidad. A continuación se enumeran una serie de causas posibles de $\mathrm{NO}$ asistencia y te pedimos que nos indiques si han influido en tu decisión de no asistir — de 0 (NADA) a 4 (MUCHO)—.

\begin{tabular}{|c|c|c|c|c|c|}
\hline CAUSAS & $\mathbf{0}$ & 1 & 3 & 3 & 4 \\
\hline $\begin{array}{l}\text { 1. El nivel de conocimientos necesarios para seguir la clase es } \\
\text { muy alto. }\end{array}$ & & & & & \\
\hline $\begin{array}{l}\text { 2. Crees que es más provechoso dedicar } 1 \text { hora al estudio (en la } \\
\text { Biblioteca, en casa...) que asistir a clase. }\end{array}$ & & & & & \\
\hline $\begin{array}{l}\text { 3. Suelo faltar a aquellas clases sueltas en el horario (por ejemplo } \\
\text { tengo que permanecer dos o tres horas en la facultad). }\end{array}$ & & & & & \\
\hline 4. El numero de horas es excesivo y me canso. & & & & & \\
\hline 5. Tengo la asignatura pendiente y no necesito seguir las clases. & & & & & \\
\hline $\begin{array}{l}\text { 6. El profesor normalmente hace responder a los alumnos en voz } \\
\text { alta, salir a la pizarra, etc. y eso no me gusta. }\end{array}$ & & & & & \\
\hline $\begin{array}{l}\text { 7. La actuación del profesor es correcta, pero consideras que la } \\
\text { asignatura se sigue fácilmente sin sus explicaciones. }\end{array}$ & & & & & \\
\hline $\begin{array}{l}\text { 8. El nivel de conocimientos necesarios para seguir la clase es } \\
\text { muy bajo. }\end{array}$ & & & & & \\
\hline 9. El profesor no explica de manera clara y estructurada. & & & & & \\
\hline 10. Suelo no asistir a clase los días antes de un examen. & & & & & \\
\hline $\begin{array}{l}\text { 11. Necesitas descansar alguna que otra hora, y por ejemplo, acudes } \\
\text { a la Cafetería. }\end{array}$ & & & & & \\
\hline $\begin{array}{l}\text { 12. La asignatura (por ejemplo de Libre Elección) se imparte en } \\
\text { otro centro }\end{array}$ & & & & & \\
\hline 13. Dispones de apuntes de otros compañeros. & & & & & \\
\hline 14. La clase me coincide con otras asignaturas teóricas. & & & & & \\
\hline
\end{tabular}




\begin{tabular}{|c|c|c|c|c|c|}
\hline CAUSAS & $\mathbf{0}$ & 1 & 3 & 3 & 4 \\
\hline 15. Aprovecho la hora para asistir a tutoría. & & & & & \\
\hline $\begin{array}{l}\text { 16. El material proporcionado por el profesor (transparencias, } \\
\text { apuntes, ejercicios, etc.) me permite preparar la asignatura sin } \\
\text { asistir a las clases. }\end{array}$ & & & & & \\
\hline 17. Mi grupo de amigos me anima a no asistir a clase. & & & & & \\
\hline 18. No sé tomar apuntes. & & & & & \\
\hline $\begin{array}{l}\text { 19. He faltado por algún motivo (como enfermedad, por ejemplo) } \\
\text { durante varios días y ya considero que he perdido "el hilo". }\end{array}$ & & & & & \\
\hline 20. La clase me coincide con prácticas de otras asignaturas. & & & & & \\
\hline $\begin{array}{l}\text { 21. La ausencia suele ocurrir un día concreto (por ejemplo lunes o } \\
\text { viernes) }\end{array}$ & & & & & \\
\hline 22. Las clases resultan aburridas & & & & & \\
\hline $\begin{array}{l}\text { 23. Falta involuntaria por problemas de transporte (resulta caro o es } \\
\text { incompatible con tu horario). }\end{array}$ & & & & & \\
\hline $\begin{array}{l}\text { 24. Dejo alguna asignatura porque me he dado cuenta de que me he } \\
\text { matriculado de más asignaturas de las que puedo aprobar. }\end{array}$ & & & & & \\
\hline $\begin{array}{l}\text { 25. Sueles faltar a las clases peor situadas en el horario: coinciden } \\
\text { con la hora del almuerzo. }\end{array}$ & & & & & \\
\hline $\begin{array}{l}\text { 26. La asignatura tiene fama de difícil y me han recomendado no ir, } \\
\text { porque no merece la pena. }\end{array}$ & & & & & \\
\hline $\begin{array}{l}\text { 27. Sueles faltar a las clases peor situadas en el horario: primera } \\
\text { hora de la mañana o última hora de la tarde. }\end{array}$ & & & & & \\
\hline $\begin{array}{l}\text { 28. No puedes asistir a clase por tu trabajo o por esta realizando el } \\
\text { servicio militar o la prestación social sustitutoria. }\end{array}$ & & & & & \\
\hline $\begin{array}{l}\text { 29. La asignatura me resulta fácil y puedo aprobarla sin asistir a } \\
\text { clase. }\end{array}$ & & & & & \\
\hline $\begin{array}{l}\text { 30. Dejo de asistir en fechas concretas (vísperas de periodos de } \\
\text { exámenes, vacacionales, etc.) }\end{array}$ & & & & & \\
\hline $\begin{array}{l}\text { 31. El material didáctico de apoyo que utiliza el profesor no es } \\
\text { adecuado. }\end{array}$ & & & & & \\
\hline $\begin{array}{l}\text { 32. Estoy matriculado en esta titulación, pero realmente no me } \\
\text { gusta. }\end{array}$ & & & & & \\
\hline 33. Otros (especificar) & & & & & \\
\hline
\end{tabular}




\section{RESUMEN}

Ordena los tres grandes bloques de posibles causas de falta de asistencia a clase, en tu caso, por orden de importancia, de 1 (MÁS IMPORTANTE) a 3 (MENOS IMPORTAN$\mathrm{TE})$ :

- DECISIÓN PERSONAL. Consideras como alumno que las clases no te resultan provechosas y prefieres emplear tu tiempo de otra forma

- DIFICULTADES EXTERNAS. Desearía asistir a clase pero me es muy difícil por motivos de horario, transporte, etc.

- DIFICULTADES DE TIPO PERSONAL. El alumno tiene obligaciones laborales o familiares que atender.

\section{PROPUESTAS}

Proponga medidas que en su caso mejorarían su nivel de asistencia.

1.

2.

3. 